\title{
AVANCES Y RETOS DE LA REVISTA PERUANA DE MEDICINA EXPERIMENTAL Y SALUD PÚBLICA AL 2010
}

\author{
[ADVANCES AND CHALLENGES OF THE REVISTA PERUANA DE \\ MEDICINA EXPERIMENTAL Y SALUD PÚBLICA IN THE YEAR 2010]
}

\author{
Zuño Burstein ${ }^{1}$, César Cabezas²
}

Tal como lo expresáramos en el editorial de esta Revista en el primer número del año 2009, uno de los objetivos que nos habíamos trazado era lograr, durante ese año, incluso dispuesto así como meta por la actual Jefatura del Instituto Nacional de Salud (INS), que nuestra Revista reuniera todos los exigentes requisitos para su indización en las bases de datos de mayor prestigio a nivel mundial, mediante el aseguramiento de evidenciar una altísima calidad en su contenido y la obligada periodicidad en su edición, garantizando a los autores que publican con nosotros, que sus artículos serían acreditados y leídos a nivel mundial y obtener así, la calificación externa de publicación médico-científica de excelencia (1).

La Revista Peruana de Medicina Experimental y Salud Pública inicia su publicación el año 1942, con el volumen 1, números 1 y 2, editada por los doctores Telémaco Battistini (2) y Carlos Gutiérrez Noriega (3), con el nombre de Revista de Medicina Experimental, como órgano oficial de difusión científica del Instituto de Higiene y Salud Pública, se publicó trimestralmente hasta 1960, luego de lo cual tuvo dificultades para su continuidad. En 1997 se reinicia la publicación como "Segunda Época" y en el año 2002 cambia su nombre al que lleva en la actualidad, es decir, son doce años de publicación regular, con un progresivo mejoramiento de la calidad ${ }^{(4)}$, habiendo logrado reunir durante el año 2009 todos los requisitos para aplicar a Medline, que es una de las bases de datos más exigente para las publicaciones médico-científicas a nivel mundial y en la que ha sido aceptada, hasta el momento, sólo una revista peruana. Actualmente, estamos incorporados a catorce sistemas de indización y bases de datos a nivel nacional, regional e internacional (AGORA, DOAJ, HINARI, IMBIOMED, IndexCopernicus, LATINDEX, LILACS, LIPECS, MedicLatina, OARE, RedALyC, REPIDISCA, SciELO Perú y SISBIB-UNMSM) y la consulta electrónica de nuestra publicación superó el millón y medio de descargas de artículos durante el 2009 sumando sólo tres bases de datos y la consulta en nuestra página web: www.ins.gob. pe/rpmesp.

Cabe resaltar que en las evaluaciones bibliométricas realizadas por investigadores externos, nos reconocen como la revista médica peruana que publica más artículos y más investigaciones ${ }^{(5)}$, ello ha motivado que se nos catalogue como la mejor revista médica peruana en la actualidad ${ }^{(6)}$. Estos logros se han obtenido por la rigurosidad establecida, tanto en la periodicidad de su publicación como en su presentación formal y, sobre todo, por la calidad de su contenido, que está orientado específicamente al enfoque de los problemas de salud pública de interés para nuestro país, con investigaciones e información trascendente de procedencia nacional y extranjera, destacando en la proporción que le corresponde los aspectos que refuerzan nuestra identidad nacional. Con ese fin, se dedica un espacio a una sección de Personalidades Destacadas de la Salud Pública en el Perú, se defiende editorialmente el nombre de Enfermedad de Carrión para la Bartonelosis humana, producida por la Bartonella baciliformis (7), y se asume la actitud que corresponde a nuestra Revista, ejemplarizando, en la medida de nuestras posibilidades, en los aspectos éticos de las publicaciones médico-científicas de nuestro país ${ }^{\left({ }^{8}\right)}$. Cumplimos, así, con la función referencial que corresponde al INS como brazo científico del Ministerio de Salud, organismo rector de la salud pública en el Perú.

La Revista Peruana de Medicina Experimental y Salud Pública ha abierto sus páginas a colaboradores nacionales y extranjeros, tanto del sector público como privado, para que publiquen sus investigaciones

\footnotetext{
Director, Revista Peruana de Medicina Experimental y Salud Pública, Instituto Nacional de Salud. Lima, Perú.

Editor General, Revista Peruana de Medicina Experimental y Salud Pública, Instituto Nacional de Salud. Lima, Perú.

Correo electrónico: zburstein@ins.gob.pe; ccabezas@ins.gob.pe
} 
realizadas individual o colectivamente en centros de investigación de instituciones universitarias, en el Ministerio de Salud, como en nuestro Instituto Nacional de Salud, en servicios de salud, hospitalarios y de otro origen; pero, para todos ellos, sin discriminación alguna, se exige que se ajusten, para la aceptación de sus artículos, a las normas generales y específicas establecidas en las instrucciones para los autores de la Revista para cada tipo de artículo, ya sea bajo la forma de artículos originales de investigación, artículos de revisión, contribución a los simposios ${ }^{(9)}$, sección de Personalidades Destacadas de la Salud Pública en el Perú, sección especial, reporte de casos, galería fotográfica o cartas al editor, que constituyen las diferentes secciones de la revista; las que se ajustan a la normatividad establecida internacionalmente por el Comité Internacional de Editores de Revistas Médicas.

Los artículos presentados a nuestra Revista, para ser registrados, deben cumplir con los requerimientos formales establecidos y, una vez verificada su conformidad, son presentados al Comité Editor, quienes toman la decisión final de su aceptación, esta aceptación toma en cuenta la pertinencia de su publicación, de acuerdo con la política editorial de la Revista y establece su ubicación en la sección correspondiente. Una vez aceptado, se comunica tal decisión al autor y el artículo se envía a la revisión por pares, quienes, en forma anónima, revisan, califican y plantean las sugerencias pertinentes. La designación de revisores se hace de acuerdo con la especialidad requerida para cada caso; para los artículos de investigación originales se compromete la participación de dos a más revisores ajenos a los autores que, habitualmente, son destacadas personalidades científicas, nacionales o extranjeras, con experiencia en publicación, quienes son comprometidos ad honórem y que, muy cordialmente, aceptan nuestro requerimiento; al final de cada volumen anual, la Revista agradece públicamente la participación de esas destacadas personalidades, sin mencionar en qué artículo participaron.

Los artículos calificados y con las sugerencias pertinentes regresan al Comité Editor para su aprobación o desaprobación; en caso de observaciones mayores, se encarga a los editores científicos (asociado y adjunto) que trabajen con los autores para lograr su perfeccionamiento y se vuelven a presentar al Comité Editor para la decisión final y establecer, si es aprobado, la sección y el número donde será publicado, lo que es comunicado al autor.

Todo este trámite y filtros establecidos garantizan la idoneidad y seriedad del proceso, que culmina con un artículo que, bajo la responsabilidad del autor en su contenido, es publicado con la calificación aprobatoria de expertos en el tema. Todo el proceso para los artículos originales de investigación toma en promedio unos tres meses de tiempo y un lapso menor, con menor número de revisores, para las otras secciones.

Tal como lo establecimos en el Editorial del primer número del Volumen 26, correspondiente al año 2009 (1), se fijó para cada número del Volumen, cuatro en total, un tema de importancia y actualidad de salud pública, comprometiendo como Editores Invitados a destacados profesionales de nuestro medio, que convocaron, en coordinación con el Director y los Editores Científicos de la Revista, a especialistas nacionales o extranjeros para colaborar en su tema, y se ocuparon, en forma especial, de la sección Simposio, que representa una tribuna abierta para confrontar planteamientos que, asumidos por sus autores, permitan una visualización integral de la problemática planteada. Este proyecto resultó un éxito y todas las contribuciones, igualmente calificadas de acuerdo con las exigencias normativas de la Revista, cumplieron con el objetivo previsto, constituyéndose en un valioso aporte informativo para todos los interesados del país y del exterior.

Agradecemos la valiosa participación de los siguientes profesionales que se ocuparon exitosamente, con gran entusiasmo y responsabilidad, de los temas a su cargo: al Dr. Hugo Rengifo Cuéllar, Médico Cirujano, especialista en Inmunología y Alergia; politólogo y planificador en salud; Presidente de la Sociedad Peruana de Salud Ambiental (SOPERSA); Representante de la Sociedad Civil ante el Comité Nacional de Salud Ambiental del Consejo Nacional de Salud del MINSA, que tuvo a su cargo, como Editor Invitado, el tema Salud Ambiental, publicado en dos números de la Revista. Al Dr. Anibal Velásquez Valdivia, Médico con maestría en epidemiología, Magister en Malariología y Saneamiento Ambiental, profesor de posgrado de la Universidad Nacional Mayor de San Marcos (UNMSM), Universidad Peruana Cayetano Heredia (UPCH), Pontificia Universidad Católica del Perú, Universidad Peruana de Ciencias Aplicadas, quien tuvo a su cargo el tema: Aseguramiento Universal en Salud. Al Dr. Eduardo Ticona Chávez, Médico especialista en Enfermedades Infecciosas y Tropicales; Doctor en Medicina; Maestro en Ciencias Tropicales Biomédicas; Profesor de las Facultades de Medicina de la UNMSM y San Martín de Porras; Miembro del Panel de Revisiones Técnicas en Tuberculosis del Fondo Global de Lucha contra el SIDA, Tuberculosis y Malaria de la OMS; Responsable Nacional del Control de Tuberculosis del MINSA 2002-2003, quien tuvo a su cargo el tema de Tuberculosis, como editor invitado. Al Dr. Pedro Alvarez-Falconí, Médico especialista en Farmacología; Doctor en Medicina; Master (c) en Ética Médica y Bioética Clínica; Bachiller en Derecho; Ex Director General del Centro Nacional de 
Control de Calidad de Medicamentos del INS/MINSA, Profesor Principal de Farmacología de la Universidad Nacional Federico Villarreal, quien tuvo a su cargo como Editor Invitado el tema Política de Medicamentos.

Para este año, el Comité Editor del INS ha programado cuatro temas de interés y actualidad de salud pública para nuestro país, para cada uno de los números del volumen correspondiente al año 2010, habiéndose ya comprometido para cada uno de ellos como Editores Invitados a los siguientes profesionales, expertos en cada uno de los temas: comenzando con este primer número, Vol. 27, N. ${ }^{\circ}$, ene-mar, con el tema Interculturalidad en Salud, a cargo del Dr. Oswaldo Salaverry García, Médico Cirujano; Doctor en Medicina; Profesor de Medicina Preventiva y Salud Pública e Historia de la Medicina en la Facultad de Medicina de San Fernando de la UNMSM; ex Presidente de la Comisión Andina de Salud Intercultural; Responsable de la Estrategia Sanitaria Nacional de Salud de los Pueblos Indígenas y Director General del Centro Nacional de Salud Intercultural del INS/MINSA. El segundo número del año tendrá como tema prioritario Accidentes de tránsito, habiéndose comprometido como Editor Invitado al Dr. J. Jaime Miranda, con Maestría y Doctorado en Epidemiología en el London School of Hygiene and Tropical Medicine; Profesor Investigador de la Facultad de Medicina de la UPCH; Investigador Principal del Programa de Investigación en Accidentes de Tránsito, financiado por el INS. El tercer número de la revista tiene como tema principal Políticas de investigación en salud, a cargo como Editor Invitado del Dr. Martín Yagui Moscoso, Médico cirujano especialista en Patología Clínica con maestría en Epidemiología Hospitalaria; Profesor del Departamento de Medicina Preventiva y Salud Pública de la UNMSM, Presidente de la Sociedad Peruana de Epidemiología y Director de la Oficina General de Investigación y Transferencia Tecnológica del INS. El cuarto y último número del año tendrá como tema principal a las Zoonosis parasitarias, a cargo como editor invitado del Dr. César Náquira Velarde, Médico Parasitólogo; Doctor en Medicina; Profesor Emérito de la UNMSM; Académico de Número de la Academia Nacional de Medicina; Profesor Principal de Parasitología de las Facultades de Medicina de la UNMSM, de la Universidad Ricardo Palma y del Curso de Parasitología de la Universidad de Alabama, USAUniversidad Peruana Cayetano Heredia; ex Jefe del Laboratorio de Zoonosis de la División de Parasitología del INS y ex Jefe del INS/MINSA.

Si bien la Revista Peruana de Medicina Experimental y Salud Pública es el órgano científico oficial del Instituto Nacional de Salud, la política editorial de la Revista es el de estar abierta a la discusión alturada de quienes escriben en ella, dentro del marco de las normas de publicación establecidas, en búsqueda de la verdad y las evidencias que puedan contribuir a mejorar la salud pública en nuestro país; por lo que en medio de la diversidad y complejidad de los temas tratados y publicados en la Revista, el contenido es de responsabilidad de los autores. Hacemos esta precisión, porque en salud pública, pueden tratarse temas controversiales en los que pueda haber puntos de vista diferentes, sobre todo en los Simposios y artículos de opinión, cuya amplia discusión no interfiere con la política institucional del INS ni la del Ministerio de Salud.

Parafinalizar, consideramos que con los Editores Invitados, cuyos créditos arriba exhibimos, tenemos garantizada la alta calidad en el desarrollo de los temas principales que abordará nuestra Revista durante el presente año, que ha contado durante el año pasado con el invalorable concurso de los distinguidos miembros del Comité Editor del INS, con el diligente apoyo administrativo y con la decisión tomada en su beneficio por la alta Dirección del INS y del MINSA para lograr que nuestra publicación sea un documento referente que prestigie a la medicina peruana en el país y en el extranjero.

\section{REFERENCIAS BIBLIOGRÁFICAS}

1. Burstein Z. La Revista Peruana de Medicina Experimental y Salud Pública en el año 2009. Rev Peru Med Exp Salud Publica. 2009; 26(1): 3-4.

2. Campos HA. Carlos Gutiérrez-Noriega, humanista y pionero de la neurofarmacología y la psiquiatría biológica en el Perú. Rev Peru Med Exp Salud Publica. 2009; 26(4): 577-79.

3. Burstein Z. Telémaco Battistini Sánchez (1895-1960). Rev Peru Med Exp Salud Publica. 2010; 27(1): 142-45.

4. Cabezas C, Mayta-Tristán P. Evolución y perspectivas de la Revista Peruana de Medicina Experimental y Salud Pública, 1945-2008. Rev Peru Med Exp Salud Publica. 2008; 25(2): 167-68.

5. Huamaní C, Pacheco-Romero J. Visibilidad y producción de las revistas biomédicas peruanas. Rev Gastroenterol Peru. 2009; 29(2): 132-39.

6. Cáceres CF, Mendoza W. Globalized research and "national science": the case of Peru. Am J Public Health. 2009; 99(10): 1792-98.

7. Burstein Z, Mayta-Tristán P. En defensa de la denominación como "Enfermedad de Carrión" para la bartonelosis humana producidad por la Bartonella bacilliformis. Rev Peru Med Exp Salud Publica. 2007; 24(2): 103-6.

8. Mayta-Tristán P, Curioso WH. Política editorial ante la detección de una publicación redundante. Rev Peru Med Exp Salud Publica. 2009; 26(1): 5-8.

9. Burstein Z. Simposios y números temáticos en la revista. Rev Peru Med Exp Salud Publica. 2008; 25(1): 5-6. 